\title{
Analysis of some ruin-related quantities in a Markov-modulated risk model
}

\author{
Jingchao $\mathrm{Li}^{1}$, David C M Dickson², Shuanming $\mathrm{Li}^{2}$ \\ ${ }^{1}$ College of Mathematics and Computer Science, Shenzhen University, \\ Nanhai Ave 3688, Shenzhen, Guangdong, P.R. China, 518060 \\ ${ }^{2}$ Centre for Actuarial Studies, Department of Economics, \\ University of Melbourne, VIC 3010, Australia
}

\begin{abstract}
In this paper, we study the joint Laplace transform and probability generating function of some random quantities that occur in each environment state by the time of ruin in a Markov-modulated risk process. These quantities include the duration spent in each state, the number of claims and the aggregate amount of claims that occurred in each state by the time of ruin.

Explicit formulae for the joint transforms, given the initial surplus, and the initial and terminal environment states, are expressed in terms of a matrix version of the scale function. Moments and covariances of these ruinrelated quantities are obtained and numerical illustrations are presented.

The joint transform of the duration spent in each state, the number of claims and the aggregate amount of claims that occurred in each state by the time when the surplus attains a certain level are also investigated.
\end{abstract}

Keywords: Markov-modulated risk model; the duration in a certain state; the number of claims and aggregate claims in a certain state; first hitting time; matrix scale function 


\section{Introduction}

Markov-modulated risk models have been studied by a number of authors since the important paper by Asmussen (1989). In such a model, both the claim amounts and the claim arrivals are influenced by an external environment process which we denote by $\{J(t) ; t \geq 0\}$. This process is assumed to be a homogeneous, irreducible and recurrent Markov process on a finite state space $E=\{1,2, \ldots, m\}$. The process has intensity matrix $\mathbf{A}=\left(\alpha_{i, j}\right)_{i, j=1}^{m}$, with $\alpha_{i, i}:=-\alpha_{i}$ for $i \in E$, and has stationary distribution $\overrightarrow{\boldsymbol{\pi}}=\left(\pi_{1}, \pi_{2}, \ldots, \pi_{m}\right)$.

Let $N(t)$ denote the number of claims occurring in $(0, t]$. If $J(s)=i$ for all $s$ in a small interval $(t, t+h]$, then $N(t+h)-N(t)$ represents the number of claims occurring in that interval, and has a Poisson distribution with parameter $\lambda_{i}(>0)$. The process $\{N(t) ; t \geq 0\}$ is a Markov-modulated Poisson process, and can also be viewed as a Poisson process with its parameter driven by the external environment process $\{J(t) ; t \geq 0\}$.

Next, we assume that given $J(t)=i$, individual claim amounts have distribution function $F_{i}$ and probability density function $f_{i}$, with Laplace transform $\hat{f}_{i}(s)=\int_{0}^{\infty} e^{-s x} f_{i}(x) d x$, and finite mean $\mu_{i}(i \in E)$. We further assume that premiums are received continuously at a positive constant rate $c_{i}$.

For $i \in E$, let $\left\{U_{i}(t)\right\}_{t \geq 0}$ be the surplus process in the classical risk model with premium rate $c_{i}$, Poisson parameter $\lambda_{i}$, and individual claim amount distribution $F_{i}$. Then the surplus process $\{U(t) ; t \geq 0\}$ in our Markov-modulated risk model is given by

$$
U(t)=u+\sum_{i=1}^{m} \int_{0}^{t} I(J(s)=i) d U_{i}(s), \quad t \geq 0,
$$

where $u \geq 0$ is the initial surplus and $I$ is the indication function. The positive loading condition is assumed to hold, i.e.,

$$
\sum_{i=1}^{m} \pi_{i}\left(c_{i}-\lambda_{i} \mu_{i}\right)>0 .
$$

We next define $T_{u}=\inf \{t \geq 0: U(t)<0\}$ to be the time of ruin, with $T_{u}=\infty$ if $U(t) \geq 0$ for all $t \geq 0$, and

$$
\psi_{i, j}(u)=\mathbb{P}\left(T_{u}<\infty, J\left(T_{u}\right)=j \mid J(0)=i\right), \quad i, j \in E,
$$

to be the ultimate ruin probability with ruin caused by a claim in environment state $j$ given initial environment state $i$, and hence

$$
\psi_{i}(u)=\sum_{j=1}^{m} \psi_{i, j}(u), \quad u \geq 0, i \in E,
$$


is the probability of ultimate ruin given initial environment state $i$.

Let $N_{u}:=N\left(T_{u}\right)$ be the number of claims and $S_{u}:=S\left(T_{u}\right)=\sum_{n=1}^{N\left(T_{u}\right)} X_{n}$ be the aggregate claims by the time of ruin, respectively, where $X_{n}$ is the amount of the $n$th claim. Now $T_{u}, N_{u}$ and $S_{u}$ can be decomposed as

$$
T_{u}=\sum_{k=1}^{m} T_{u, k}, \quad N_{u}=\sum_{k=1}^{m} N_{u, k}, \quad S_{u}=\sum_{k=1}^{m} S_{u, k}
$$

where $T_{u, k}$ is the duration that the surplus process spends in state $k \in E$ until the time of ruin, $N_{u, k}$ is the number of claims that occur in state $k$ until the time of ruin, and $S_{u, k}$ is the aggregate claims that occur in state $k$ until the time of ruin.

For $\delta_{k}, r_{k} \geq 0,0<v_{k} \leq 1$, and $k \in E$, define

$$
\phi_{i, j}(u)=\mathbb{E}\left[e^{-\sum_{k=1}^{m}\left(\delta_{k} T_{u, k}+r_{k} S_{u, k}\right)} \prod_{k=1}^{m} v_{k}^{N_{u, k}} I\left(T_{u}<\infty, J\left(T_{u}\right)=j\right) \mid J(0)=i\right]
$$

to be the joint Laplace transform and probability generating function (p.g.f.) of $T_{u, k}, S_{u, k}$ and $N_{u, k}$ for $k=1,2, \ldots, m$, with ruin caused by a claim in state $j$, given initial surplus $u$ and initial environment state $i \in E$. Then

$$
\phi_{i}(u)=\sum_{j=1}^{m} \phi_{i, j}(u), \quad u \geq 0, i \in E
$$

is the joint Laplace transform and p.g.f. of $T_{u, k}, S_{u, k}$, and $N_{u, k}$ for $k=1,2, \ldots, m$, given initial surplus $u$ and initial environment state $i \in E$.

The function $\phi_{i, j}(u)$ defined in (1.2) gives a unified treatment of $T_{u, k}, N_{u, k}$, and $S_{u, k}$, for $k=1,2, \ldots, m$, and many special cases can be obtained by specifying parameters. Some examples are as follows.

- If $\delta_{k}=\delta, r_{k}=r$ and $v_{k}=v$ for $k \in E$, then

$$
\phi_{i, j}(u)=\mathbb{E}\left[e^{-\delta T_{u}-r S_{u}} v^{N_{u}} I\left(T_{u}<\infty, J\left(T_{u}\right)=j\right) \mid J(0)=i\right]
$$

is the joint Laplace transform and p.g.f of $T_{u}, S_{u}$ and $N_{u}$.

- If $\delta_{k}>0, r_{k}>0,0<v_{k}<1, \delta_{l}=0, r_{l}=0, v_{l}=1$ for $l \neq k$, then

$$
\phi_{i, j}(u)=\mathbb{E}\left[e^{-\delta_{k} T_{u, k}-r_{k} S_{u, k}} v_{k}^{N_{u, k}} I\left(T_{u}<\infty, J\left(T_{u}\right)=j\right) \mid J(0)=i\right]
$$

is the joint Laplace transform and p.g.f. of $T_{u, k}, S_{u, k}$ and $N_{u, k}$ for a particular state $k$. Further setting $v_{k}=1$ and $r_{k}=0, \phi_{i, j}(u)$ gives the Laplace transform of $T_{u, k}$; setting $\delta_{k}=r_{k}=0, \phi_{i, j}(u)$ gives the p.g.f. of $N_{u, k}$; and setting $\delta_{k}=0$ and $v_{k}=1, \phi_{i, j}(u)$ gives the Laplace transform of $S_{u, k}$. 
- If $\delta_{k}>0, \delta_{l}>0, \delta_{i}=0, i \neq k, i \neq l, r_{i}=0, v_{i}=1$, for $i \in E$, then

$$
\phi_{i, j}(u)=\mathbb{E}\left[e^{-\delta_{k} T_{u, k}-\delta_{l} T_{u, l}} I\left(T_{u}<\infty, J\left(T_{u}\right)=j\right) \mid J(0)=i\right]
$$

is the joint Laplace transform of $T_{u, k}$ and $T_{u, l}$. Similarly, the joint Laplace transform of $S_{u, k}$ and $S_{u, l}$ and the joint p.g.f. of $N_{u, k}$ and $N_{u, l}$ can also be obtained.

There has been much written about the Markov-modulated risk model in recent times. References include Asmussen et al. (1995), Bäuerle (1996), Schmidli (1997), Snoussi (2002), Lu and Li (2005), Lu (2006), Ng and Yang (2006), Li and $\mathrm{Lu}$ (2007, 2008), and $\mathrm{Li}$ et al. (2015). An excellent review paper of the Markov-modulated Poisson process is Fischer and Meier-Hellstern (1993). In this paper, we investigate the joint transform, moments and covariances of $T_{u, k}, N_{u, k}$ and $S_{u, k}$ for $k=1,2, \ldots, m$. Section 2 gives some preliminaries used to derive our main results, then a matrix form integro-differential equation is derived in Section 3. Explicit expressions for $\phi_{i, j}(0)$ are given in Section 4, and in Section 5 , we study $\phi_{i, j}(u)$ for $u>0$. A recursive formula for the $n$-th moment of $T_{u, k}$ is derived in Section 6. In Section 7, we give some numerical examples on moments and covariances involving $T_{u, k}$ and $N_{u, k}$. In Section 8, we give a compact matrix form expression for the joint transform of the duration, number of claims, and the aggregate claims in each state by the first hitting time of a level $b \geq u$.

\section{Preliminaries}

The operator

$$
\mathcal{T}_{s} f(x)=\int_{x}^{\infty} e^{-s(y-x)} f(y) d y, \quad x \geq 0,
$$

where $f$ is an integrable real function was introduced by Dickson and Hipp (2001) for $s \in \mathbb{R}$ and extended by Li and Garrido (2004) to cover $s \in \mathbb{C}$. In particular, $\mathcal{T}_{s} f(0)=\hat{f}(s)$ is the Laplace transform of $f$.

In this paper, the following operator is needed to simplify derivations of our main results:

$$
\mathcal{T}_{\mathbf{s}} \mathbf{f}(x)=\int_{x}^{\infty} e^{-\mathbf{s}(y-x)} \mathbf{f}(y) d y=\operatorname{diag}\left(\mathcal{T}_{s_{1}} f_{1}(x), \ldots, \mathcal{T}_{s_{m}} f_{m}(x)\right),
$$

where $\mathbf{s}=\operatorname{diag}\left(s_{1}, s_{2}, \ldots, s_{m}\right)$ and $\mathbf{f}(x)=\operatorname{diag}\left(f_{1}(x), f_{2}(x), \ldots, f_{m}(x)\right)$. Clearly,

$$
\mathcal{T}_{\mathbf{s}_{1}} \mathcal{T}_{\mathbf{s}_{2}} \mathbf{f}(x)=\left(\mathbf{s}_{1}-\mathbf{s}_{2}\right)^{-1}\left[\mathcal{T}_{\mathbf{s}_{2}} \mathbf{f}(x)-\mathcal{T}_{\mathbf{s}_{1}} \mathbf{f}(x)\right], \quad x \geq 0
$$


We remark that $\mathcal{T}_{\mathbf{s}_{1}} \mathcal{T}_{\mathbf{s}_{2}} \mathbf{f}(x)$ exists if the inverse of the diagonal matrix $\mathbf{s}_{1}-\mathbf{s}_{2}$ exists. However, it may also exist when this inverse does not exist. For example, if $\mathbf{s}_{1}=\mathbf{s}_{2}=\mathbf{s}$ then $\mathcal{T}_{\mathbf{s}_{1}} \mathcal{T}_{\mathbf{s}_{2}} \mathbf{f}(x)$ is a diagonal matrix with element $(i, i)$ equal to $-\frac{d}{d s_{i}} T_{s_{i}} f_{i}(x)$ for $i \in E$.

\section{Remarks}

1. Li and $\mathrm{Lu}(2008)$ extend the Dickson-Hipp operator to an integrable realvalued matrix function $\mathbf{B}$ with definition:

$$
\mathcal{T}_{s} \mathbf{B}(x)=\int_{x}^{\infty} e^{-s(y-x)} \mathbf{B}(y) d y, \quad s \in \mathbb{C}, x \geq 0 .
$$

2. Feng (2009) extends the Dickson-Hipp operator to the following matrix version:

$$
\mathcal{T}_{\mathbf{S}} f(x)=\int_{x}^{\infty} e^{-\mathbf{S}(y-x)} f(y) d y, \quad x \geq 0,
$$

where $\mathbf{S}$ is a square matrix such that the above integral exists.

Now we define the divided differences of a matrix $\mathbf{A}(s)$, with respect to distinct numbers $s_{1}, s_{2}, \ldots$, recursively, as follows:

$$
\begin{aligned}
\mathbf{A}\left[s_{1}, s\right] & =\frac{\mathbf{A}(s)-\mathbf{A}\left(s_{1}\right)}{s-s_{1}}, \\
\mathbf{A}\left[s_{1}, s_{2}, s\right] & =\frac{\mathbf{A}\left[s_{1}, s\right]-\mathbf{A}\left[s_{1}, s_{2}\right]}{s-s_{2}}, \mathbf{A}\left[s_{1}, s_{2}, s_{3}\right] \\
\mathbf{A}\left[s_{1}, s_{2}, s_{3}, s\right] & =\frac{\mathbf{A}\left[s_{1}, s_{2}, s\right]-s_{3}}{s-s_{3}}
\end{aligned}
$$

and so on. As for the divided differences of a function (Gerber and Shiu (2005)), we have the following formula for the $(k-1)$ th divided difference

$$
\mathbf{A}\left[s_{1}, s_{2}, \ldots, s_{k}\right]=\sum_{j=1}^{k} \frac{\mathbf{A}\left(s_{j}\right)}{\prod_{i=1, i \neq j}^{k}\left(s_{j}-s_{i}\right)} .
$$

\section{A system of integro-differential equations}

Using the same arguments as in Ng and Yang (2006) or Li and Lu (2008), we obtain the following integro-differential equations for $\phi_{i, j}(u)$ by conditioning on the events occurring in a small time interval $[0, h]$, for $i \in E$ :

$$
c_{i} \phi_{i, i}^{\prime}(u)=\left(\lambda_{i}+\delta_{i}\right) \phi_{i, i}(u)-\sum_{k=1}^{m} \alpha_{i, k} \phi_{k, i}(u)
$$




$$
\begin{aligned}
& -v_{i} \lambda_{i}\left[\int_{0}^{u} \phi_{i, i}(u-x) e^{-r_{i} x} f_{i}(x) d x+\int_{u}^{\infty} e^{-r_{i} x} f_{i}(x)\right], \\
c_{i} \phi_{i, j}^{\prime}(u)= & \left(\lambda_{i}+\delta_{i}\right) \phi_{i, j}(u)-v_{i} \lambda_{i} \int_{0}^{u} \phi_{i, j}(u-x) e^{-r_{i} x} f_{i}(x) d x \\
& -\sum_{k=1}^{m} \alpha_{i, k} \phi_{k, j}(u) .
\end{aligned}
$$

We now introduce the following notation for matrices:

$$
\begin{aligned}
\mathbf{c} & =\operatorname{diag}\left(c_{1}, c_{2}, \ldots, c_{m}\right), \\
\boldsymbol{\lambda} & =\operatorname{diag}\left(\lambda_{1}, \lambda_{2}, \ldots, \lambda_{m}\right), \\
\mathbf{v} & =\operatorname{diag}\left(v_{1}, v_{2}, \ldots, v_{m}\right), \\
\mathbf{r} & =\operatorname{diag}\left(r_{1}, r_{2}, \ldots, r_{m}\right), \\
\boldsymbol{\delta} & =\operatorname{diag}\left(\delta_{1}, \delta_{2}, \ldots, \delta_{m}\right), \\
\mathbf{F}(x) & =\operatorname{diag}\left(F_{1}(x), F_{2}(x), \ldots, F_{m}(x)\right), \\
\boldsymbol{\phi}(u) & =\left(\phi_{i, j}(u)\right)_{m \times m} .
\end{aligned}
$$

Then Equations (3.1) and (3.2) can be re-written in matrix form as follows:

$$
\begin{aligned}
\boldsymbol{\phi}^{\prime}(u)= & \mathbf{c}^{-1}(\boldsymbol{\lambda}+\boldsymbol{\delta}-\mathbf{A}) \boldsymbol{\phi}(u) \\
& -\mathbf{c}^{-1} \boldsymbol{\lambda} \mathbf{v}\left[\int_{0}^{u} e^{-\mathbf{r} x} \mathbf{f}(x) \boldsymbol{\phi}(u-x) d x+\int_{u}^{\infty} e^{-\mathbf{r} x} \mathbf{f}(x) d x\right] .
\end{aligned}
$$

Now let $\mathbf{f}_{\mathbf{r}}(x)=e^{-\mathbf{r} x} \mathbf{f}(x)$ with Laplace transform

$$
\hat{\mathbf{f}}_{\mathbf{r}}(s)=\int_{0}^{\infty} e^{-s x} \mathbf{f}_{\mathbf{r}}(x) d x=\int_{0}^{\infty} e^{-(s \mathbf{I}+\mathbf{r}) x} \mathbf{f}(x) d x=\mathcal{T}_{s \mathbf{I}+\mathbf{r}} \mathbf{f}(0),
$$

where $\mathbf{I}$ is the $m \times m$ identity matrix. Denote $\hat{\boldsymbol{\phi}}(s)=\int_{0}^{\infty} e^{-s u} \boldsymbol{\phi}(u) d u$ and let

$$
\mathbf{B}(s)=s \mathbf{I}-\mathbf{c}^{-1}(\boldsymbol{\lambda}+\boldsymbol{\delta}-\mathbf{A})+\mathbf{c}^{-1} \boldsymbol{\lambda} \mathbf{v} \hat{\mathbf{f}}_{\mathbf{r}}(s) .
$$

Taking the Laplace transform of Equation (3.3) we obtain

$$
\mathbf{B}(s) \hat{\boldsymbol{\phi}}(s)=\boldsymbol{\phi}(0)+\mathbf{c}^{-1} \boldsymbol{\lambda} \mathbf{v} \frac{\hat{\mathbf{f}}_{\mathbf{r}}(s)-\hat{\mathbf{f}}_{\mathbf{r}}(0)}{s}=\boldsymbol{\phi}(0)+\mathbf{c}^{-1} \boldsymbol{\lambda} \mathbf{v} \hat{\mathbf{f}}_{\mathbf{r}}[s, 0],
$$

which implies that

$$
\hat{\boldsymbol{\phi}}(s)=\frac{\mathbf{B}^{*}(s)\left[\boldsymbol{\phi}(0)+\mathbf{c}^{-1} \boldsymbol{\lambda} \mathbf{v} \hat{\mathbf{f}}_{\mathbf{r}}[s, 0]\right]}{\operatorname{Det}[\mathbf{B}(s)]}
$$


where $\mathbf{B}^{*}(s)$ is the adjoint matrix of $\mathbf{B}(s)$ and

$$
\hat{\mathbf{f}}_{\mathbf{r}}\left[s_{1}, s_{2}\right]=\frac{\hat{\mathbf{f}}_{\mathbf{r}}\left(s_{1}\right)-\hat{\mathbf{f}}_{\mathbf{r}}\left(s_{2}\right)}{s_{1}-s_{2}}=\mathcal{T}_{s_{1} \mathbf{I}+\mathbf{r}} \mathcal{T}_{s_{2} \mathbf{I}+\mathbf{r}} \mathbf{f}(0), \quad s_{1} \neq s_{2},
$$

is the divided difference of the matrix $\hat{\mathbf{f}}_{\mathbf{r}}(s)$.

\section{Explicit expressions for $\phi(0)$}

Using the matrix generalization of Rouche's Theorem (De Smit (1983)) or similar arguments to Li and Ren (2013), we can show that the generalized Lundberg equation $\operatorname{det}[\mathbf{B}(s)]=0$ has exactly $m$ roots with positive real parts, say

$$
\rho_{i}=\rho_{i}\left(\delta_{1}, \ldots, \delta_{m}, r_{1}, \ldots, r_{m}, v_{1}, \ldots, v_{m}\right), \quad i=1,2, \ldots, m .
$$

The quantities $\rho_{1}, \rho_{2}, \ldots, \rho_{m}$ play an important role in determining the initial values $\phi(0)$ and we assume that they are distinct in the sequel.

We use a similar idea to Ren (2007) to find $\boldsymbol{\phi}(0)$. Let row vector $\overrightarrow{\mathbf{q}}_{i}$ be a left eigenvector of $\mathbf{B}\left(\rho_{i}\right)$ with respect to eigenvalue 0 , i.e., $\overrightarrow{\mathbf{q}}_{i} \mathbf{B}\left(\rho_{i}\right)=\overrightarrow{\mathbf{0}}, i=1,2, \ldots, m$. Setting $s=\rho_{i}$ and left-multiplying both sides of (3.5) by the vector $\overrightarrow{\mathbf{q}}_{i}$, we have

$$
\begin{aligned}
\overrightarrow{\mathbf{0}}= & \overrightarrow{\mathbf{q}}_{i} \mathbf{B}\left(\rho_{i}\right) \hat{\boldsymbol{\phi}}\left(\rho_{i}\right)=\overrightarrow{\mathbf{q}}_{i} \boldsymbol{\phi}(0)+\overrightarrow{\mathbf{q}}_{i} \frac{1}{\rho_{i}} \int_{0}^{\infty} e^{-\rho_{i} x} e^{-\mathbf{r} x} \mathbf{f}(x) d x \mathbf{c}^{-1} \boldsymbol{\lambda} \mathbf{v} \\
& -\overrightarrow{\mathbf{q}}_{i} \frac{1}{\rho_{i}} \int_{0}^{\infty} e^{-\mathbf{r} x} \mathbf{f}(x) d x \mathbf{c}^{-1} \boldsymbol{\lambda} \mathbf{v}, \quad i=1,2, \ldots, m .
\end{aligned}
$$

In matrix form we have

$$
\begin{aligned}
\mathbf{Q} \phi(0)= & \boldsymbol{\rho}^{-1} \mathbf{Q} \int_{0}^{\infty} e^{-\mathbf{r} x} \mathbf{f}(x) d x \mathbf{c}^{-1} \boldsymbol{\lambda} \mathbf{v} \\
& -\boldsymbol{\rho}^{-1} \int_{0}^{\infty} e^{-\boldsymbol{\rho} x} \mathbf{Q} e^{-\mathbf{r} x} \mathbf{f}(x) d x \mathbf{c}^{-1} \boldsymbol{\lambda} \mathbf{v}
\end{aligned}
$$

where $\mathbf{Q}=\left(\overrightarrow{\mathbf{q}}_{1}^{\top}, \overrightarrow{\mathbf{q}}_{2}^{\top}, \ldots, \overrightarrow{\mathbf{q}}_{m}^{\top}\right)^{\top}$ and $\boldsymbol{\rho}=\operatorname{diag}\left(\rho_{1}, \rho_{2}, \ldots, \rho_{m}\right)$. As we assume that $\rho_{1}, \rho_{2}, \ldots, \rho_{m}$ are distinct, $\overrightarrow{\mathbf{q}}_{1}, \overrightarrow{\mathbf{q}}_{2}, \ldots, \overrightarrow{\mathbf{q}}_{m}$ are linearly independent and so $\mathbf{Q}$ is invertible. Then we have

$$
\begin{aligned}
\phi(0)= & \mathbf{Q}^{-1} \boldsymbol{\rho}^{-1} \mathbf{Q} \int_{0}^{\infty} e^{-\mathbf{r} x} \mathbf{f}(x) d x \mathbf{c}^{-1} \boldsymbol{\lambda} \mathbf{v} \\
& -\mathbf{Q}^{-1} \boldsymbol{\rho}^{-1} \mathbf{Q} \int_{0}^{\infty} \mathbf{Q}^{-1} e^{-\boldsymbol{\rho} x} \mathbf{Q} e^{-\mathbf{r} x} \mathbf{f}(x) d x \mathbf{c}^{-1} \boldsymbol{\lambda} \mathbf{v}
\end{aligned}
$$




$$
=\boldsymbol{\Theta}^{-1}\left[\int_{0}^{\infty} e^{-\mathbf{r} x} \mathbf{f}(x) d x-\int_{0}^{\infty} e^{-\boldsymbol{\Theta} x} e^{-\mathbf{r} x} \mathbf{f}(x) d x\right] \mathbf{c}^{-1} \boldsymbol{\lambda} \mathbf{v}
$$

where $\boldsymbol{\Theta}=\mathbf{Q}^{-1} \boldsymbol{\rho} \mathbf{Q}$. In particular, if $\mathbf{r}=\operatorname{diag}\left(r_{1}, r_{2}, \ldots, r_{m}\right)=\mathbf{0}$, then

$$
\boldsymbol{\phi}(0)=\mathbf{\Theta}^{-1}\left[\mathbf{I}-\int_{0}^{\infty} e^{-\boldsymbol{\Theta} x} \mathbf{f}(x) d x\right] \mathbf{c}^{-1} \boldsymbol{\lambda} \mathbf{v}
$$

Formula (4.1) gives a compact matrix-form expression for $\phi(0)$. Further, using a similar method to $\mathrm{Li}$ and $\mathrm{Lu}$ (2008), we can derive an alternative expression for $\phi(0)$.

For distinct $\rho_{1}, \rho_{2}, \ldots, \rho_{m}$, since $\hat{\phi}_{i, j}(s)$ is finite for $\Re(s) \geq 0$, then

$$
\mathbf{B}^{*}\left(\rho_{i}\right) \boldsymbol{\phi}(0)=-\mathbf{B}^{*}\left(\rho_{i}\right) \mathbf{c}^{-1} \boldsymbol{\lambda} \mathbf{v} \hat{\mathbf{f}}_{\mathbf{r}}\left[\rho_{i}, 0\right], \quad i=1,2, \ldots, m .
$$

Therefore

$$
\mathbf{B}^{*}\left[\rho_{1}, \rho_{2}\right] \boldsymbol{\phi}(0)=-\left(\mathbf{B}^{*} \mathbf{c}^{-1} \boldsymbol{\lambda} \mathbf{v} \hat{\mathbf{f}}_{\mathbf{r}}[\cdot, 0]\right)\left[\rho_{1}, \rho_{2}\right],
$$

where $\left(\mathbf{B}^{*} \mathbf{c}^{-1} \boldsymbol{\lambda} \mathbf{v} \hat{\mathbf{f}}_{\mathbf{r}}[\cdot, 0]\right)\left[\rho_{1}, \rho_{2}\right]$ is the divided difference of the product of matrices $\mathbf{B}^{*}(s)$ and $\mathbf{c}^{-1} \boldsymbol{\lambda} \mathbf{v} \hat{\mathbf{f}}_{\mathbf{r}}[s, 0]$ with respect to $\rho_{1}$ and $\rho_{2}$, given by

$$
\left(\mathbf{B}^{*} \mathbf{c}^{-1} \boldsymbol{\lambda} \mathbf{v} \hat{\mathbf{f}}_{\mathbf{r}}[\cdot, 0]\right)\left[\rho_{1}, \rho_{2}\right]=\mathbf{B}^{*}\left(\rho_{1}\right) \mathbf{c}^{-1} \boldsymbol{\lambda} \mathbf{v} \hat{\mathbf{f}}_{\mathbf{r}}\left[\rho_{1}, \rho_{2}, 0\right]+\mathbf{B}^{*}\left[\rho_{1}, \rho_{2}\right] \mathbf{c}^{-1} \boldsymbol{\lambda} \mathbf{v} \hat{\mathbf{f}}_{\mathbf{r}}\left[\rho_{2}, 0\right] .
$$

Recursively, we have

$$
\begin{aligned}
\mathbf{B}^{*}\left[\rho_{1}, \rho_{2}, \ldots, \rho_{m}\right] \boldsymbol{\phi}(0) & =-\left(\mathbf{B}^{*} \mathbf{c}^{-1} \boldsymbol{\lambda} \mathbf{v} \hat{\mathbf{f}}_{\mathbf{r}}[\cdot, 0]\right)\left[\rho_{1}, \rho_{2}, \ldots, \rho_{m}\right] \\
& =-\sum_{i=1}^{m} \mathbf{B}^{*}\left[\rho_{1}, \ldots, \rho_{i}\right] \mathbf{c}^{-1} \boldsymbol{\lambda} \mathbf{v} \hat{\mathbf{f}}_{\mathbf{r}}\left[\rho_{i}, \ldots, \rho_{m}, 0\right]
\end{aligned}
$$

Then we have the following result for $\phi(0)$ :

$$
\begin{aligned}
\phi(0)= & -\mathbf{c}^{-1} \boldsymbol{\lambda} \mathbf{v} \hat{\mathbf{f}}_{\mathbf{r}}\left[\rho_{m}, 0\right]-\left(\mathbf{B}^{*}\left[\rho_{1}, \rho_{2}, \ldots, \rho_{m}\right]\right)^{-1} \times \\
& \sum_{i=1}^{m-1} \mathbf{B}^{*}\left[\rho_{1}, \ldots, \rho_{i}\right] \mathbf{c}^{-1} \boldsymbol{\lambda} \mathbf{v} \hat{\mathbf{f}}_{\mathbf{r}}\left[\rho_{i}, \ldots, \rho_{m}, 0\right]
\end{aligned}
$$

\section{Explicit expressions for $\phi(u)$}

In this section, we find explicit expressions for $\phi(u)$. We first consider the case when claim amounts follow exponential distributions, and then we consider the general case by inverting the Laplace transform of $\phi(u)$. 


\subsection{Exponential claim distributions}

We assume that $f_{i}(x)=\beta_{i} e^{-\beta_{i} x}$ with $\beta_{i}>0, x>0, i \in E$, i.e., $\mathbf{f}(x)=\boldsymbol{\beta} e^{-\boldsymbol{\beta} x}$, with $\boldsymbol{\beta}=\operatorname{diag}\left(\beta_{1}, \beta_{2}, \ldots, \beta_{m}\right)$. Differentiating Equation (3.3) we obtain a 2 nd order matrix differential equation:

$\boldsymbol{\phi}^{\prime \prime}(u)+\left[\boldsymbol{\beta}+\mathbf{r}-\mathbf{c}^{-1}(\boldsymbol{\lambda}+\boldsymbol{\delta}-\mathbf{A})\right] \boldsymbol{\phi}^{\prime}(u)+\mathbf{c}^{-1}[\boldsymbol{\lambda} \mathbf{v} \boldsymbol{\beta}-(\boldsymbol{\beta}+\mathbf{r})(\boldsymbol{\lambda}+\boldsymbol{\delta}-\mathbf{A})] \boldsymbol{\phi}(u)=\mathbf{0}$,

with $\lim _{u \rightarrow \infty} \phi(u)=\mathbf{0}$ and $\lim _{u \rightarrow 0} \phi(u)=\phi(0)$. The solution to this matrix differential equation is

$$
\phi(u)=e^{-\mathbf{R} u} \phi(0),
$$

where $\mathbf{R}$ is an $m \times m$ matrix satisfying the following equation:

$$
\mathbf{R}^{2}-\left[\boldsymbol{\beta}+\mathbf{r}-\mathbf{c}^{-1}(\boldsymbol{\lambda}+\boldsymbol{\delta}-\mathbf{A})\right] \mathbf{R}+\mathbf{c}^{-1}[\boldsymbol{\lambda} \mathbf{v} \boldsymbol{\beta}-(\boldsymbol{\beta}+\mathbf{r})(\boldsymbol{\lambda}+\boldsymbol{\delta}-\mathbf{A})]=\mathbf{0} .
$$

\section{Remarks}

1. A study of approaches to solving quadratic matrix equations is conducted in Higham and Kim (2000).

2. The solution to Equation (5.1) is not unique and the one whose eigenvalues have positive real parts is our $\mathbf{R}$. This follows by consideration of the case $m=1$.

Example 5.1 Suppose that there are two states $(m=2)$ with intensity matrix:

$$
\mathbf{A}=\left(\begin{array}{cc}
-1 / 4 & 1 / 4 \\
3 / 4 & -3 / 4
\end{array}\right)
$$

with $\left(\pi_{1}, \pi_{2}\right)=(3 / 4,1 / 4)$. Further assume that $f_{1}(x)=e^{-x}, f_{2}(x)=0.5 e^{-0.5 x}$, $x>0, \lambda_{1}=1, \lambda_{2}=2 / 3, \delta_{1}=r_{1}=0.04, \delta_{2}=r_{2}=0.06, v_{1}=0.2, v_{2}=0.5$, $c_{1}=4 / 3$ and $c_{2}=5 / 3$ so that the positive loading condition holds true. Then

$$
\mathbf{R}=\left(\begin{array}{rr}
0.9774 & -0.0785 \\
0.1061 & 0.4661
\end{array}\right)
$$

To obtain this solution, we simply applied the MATLAB code in Higham and Kim (2000), and obtained the result instantaneously. 


\subsection{An explicit expression for $\phi(u)$ in terms of the matrix version of the scale function}

Define $\mathbf{W}_{\delta, \mathbf{r}, \mathbf{v}}(u)$ to be a matrix function such that

$$
\hat{\mathbf{W}}_{\boldsymbol{\delta}, \mathbf{r}, \mathbf{v}}(s)=\int_{0}^{\infty} e^{-s u} \mathbf{W}_{\boldsymbol{\delta}, \mathbf{r}, \mathbf{v}}(u) d u=[\mathbf{B}(s)]^{-1} .
$$

It follows from Equations (3.5) and (3.6) that

$$
\begin{aligned}
\hat{\boldsymbol{\phi}}(s)= & {[\mathbf{B}(s)]^{-1} \boldsymbol{\phi}(0)+\frac{1}{s}[\mathbf{B}(s)]^{-1}\left[\mathbf{B}(s)-s \mathbf{I}+\mathbf{c}^{-1}(\boldsymbol{\lambda}+\boldsymbol{\delta}-\mathbf{A})\right] } \\
& -\frac{1}{s}[\mathbf{B}(s)]^{-1} \mathbf{c}^{-1} \boldsymbol{\lambda} \mathbf{v} \hat{\mathbf{f}}_{\mathbf{r}}(0) \\
= & \frac{1}{s} \mathbf{I}-[\mathbf{B}(s)]^{-1}[\mathbf{I}-\boldsymbol{\phi}(0)]+\frac{1}{s}[\mathbf{B}(s)]^{-1}\left[\mathbf{c}^{-1}(\boldsymbol{\lambda}+\boldsymbol{\delta}-\mathbf{A})-\mathbf{c}^{-1} \boldsymbol{\lambda} \mathbf{v} \hat{\mathbf{f}}_{\mathbf{r}}(0)\right] .
\end{aligned}
$$

Inverting this Laplace transform gives

$$
\begin{aligned}
\phi(u)= & \mathbf{I}-\mathbf{W}_{\delta, \mathbf{r}, \mathbf{v}}(u)[\mathbf{I}-\boldsymbol{\phi}(0)] \\
& +\int_{0}^{u} \mathbf{W}_{\delta, \mathbf{r}, \mathbf{v}}(x) d x\left[\mathbf{c}^{-1}(\boldsymbol{\lambda}+\boldsymbol{\delta}-\mathbf{A})-\mathbf{c}^{-1} \boldsymbol{\lambda} \mathbf{v} \hat{\mathbf{f}}_{\mathbf{r}}(0)\right] .
\end{aligned}
$$

\section{Remarks}

1. $\mathbf{W}_{\delta, \mathbf{r}, \mathbf{v}}(u)$ can be viewed as the matrix version of the scale function in the Markov-modulated risk model.

2. When $m=1$, the model simplifies to the classical risk model and the result in Equation (5.2) simplifies to

$$
\begin{aligned}
\phi(u) & =\mathbb{E}\left[e^{-\delta T_{u}-r S_{u}} v^{N_{u}} I\left(T_{u}<\infty\right)\right] \\
& =1-W_{\delta, r, v}(u)[1-\phi(0)]+\frac{\lambda+\delta-\lambda v \hat{f}(r)}{c} \int_{0}^{u} W_{\delta, r, v}(x) d x,
\end{aligned}
$$

where $W_{\delta, r, v}(u)$ is a function such that

$$
\int_{0}^{\infty} e^{-s u} W_{\delta, r, v}(u) d u=\frac{c}{c s-(\lambda+\delta)+\lambda v \hat{f}(r+s)} .
$$

In particular, $W_{\delta, 0,1}(u)$ is the scale function in the classical risk model. Kyprianou (2013) points out that this scale function plays a very important role in the classical risk model and many quantities can be expressed in terms of the scale function. 
3. To evaluate $\mathbf{W}_{\boldsymbol{\delta}, \mathbf{r}, \mathbf{v}}(u)$, we need to make assumptions about the claim size distributions. If we assume that each $\hat{f}_{i}(s)$ is a rational function, then we can show that each element of $\hat{\mathbf{W}}_{\boldsymbol{\delta}, \mathbf{r}, \mathbf{v}}(s)=[\mathbf{B}(s)]^{-1}=\mathbf{B}^{*}(s) / \operatorname{Det}[\mathbf{B}(s)]$ is a rational function which is readily inverted by partial fractions. Further, the computation of $\int_{0}^{u} \mathbf{W}_{\boldsymbol{\delta}, \mathbf{r}, \mathbf{v}}(x) d x$ can be done by inverting $[\mathbf{B}(s)]^{-1} / s=$ $\mathbf{B}^{*}(s) /\{s \operatorname{Det}[\mathbf{B}(s)]\}$.

\section{The moments of $T_{u, k}$ and $T_{u}$}

Once the joint Laplace transform and p.g.f. of $T_{u, k}, N_{u, k}$ and $S_{u, k}$ has been derived, we can derive the joint and marginal moments, as well as the covariance between any two of the $3 m$ random variables. In this section, we restrict ourselves to deriving the moments of $T_{u}$ and $T_{u, k}$ for a specific $k \in E$. For $n \in \mathbb{N}$ and $i, j \in E$ we let

$$
\begin{aligned}
\psi_{i, j}^{(n)}(u) & =\mathbb{E}\left[T_{u}^{n} I\left(T_{u}<\infty, J\left(T_{u}\right)=j\right) \mid J(0)=i\right], \\
{ }_{k} \psi_{i, j}^{(n)}(u) & =\mathbb{E}\left[T_{u, k}^{n} I\left(T_{u}<\infty, J\left(T_{u}\right)=j\right) \mid J(0)=i\right]
\end{aligned}
$$

with $\psi_{i, j}^{(0)}(u)={ }_{k} \psi_{i, j}^{(0)}(u)=\psi_{i, j}(u)$.

Let $\boldsymbol{\psi}_{n}(u)=\left(\psi_{i, j}^{(n)}(u)\right)_{m \times m}$ and ${ }_{k} \boldsymbol{\psi}_{n}(u)=\left({ }_{k} \psi_{i, j}^{(n)}(u)\right)_{m \times m}$, with

$$
\boldsymbol{\psi}_{0}(u)={ }_{k} \boldsymbol{\psi}_{0}(u)=\boldsymbol{\psi}(u)=\left(\psi_{i, j}(u)\right)_{m \times m} .
$$

Let $\hat{\boldsymbol{\psi}}_{n}(s)=\left(\hat{\psi}_{i, j}^{(n)}(s)\right)_{m \times m}$ and ${ }_{k} \hat{\boldsymbol{\psi}}_{n}(s)=\left({ }_{k} \hat{\psi}_{i, j}^{(n)}(s)\right)_{m \times m}$ be their Laplace transforms with respect to $s$. It follows that

$$
\begin{aligned}
{ }_{k} \boldsymbol{\psi}_{n}(u) & =\left.(-1)^{n} \frac{\partial^{n} \boldsymbol{\phi}(u)}{\partial \delta_{k}^{n}}\right|_{\delta_{l}=r_{l}=0, v_{l}=1}, \\
{ }_{k} \hat{\boldsymbol{\psi}}_{n}(s) & =\left.(-1)^{n} \frac{\partial^{n} \hat{\boldsymbol{\phi}}(s)}{\partial \delta_{k}^{n}}\right|_{\delta_{l}=r_{l}=0, v_{l}=1}
\end{aligned}
$$

Differentiating $n$ times with respect to $\delta_{k}$ on both sides of Equation (3.5) and setting $\delta_{l}=r_{l}=0$ and $v_{l}=1$, for $l=1,2, \ldots, m$, we have

$$
\begin{aligned}
{ }_{k} \hat{\boldsymbol{\psi}}_{n}(s) & =\left[\mathbf{B}_{0}(s)\right]^{-1}\left[{ }_{k} \boldsymbol{\psi}_{n}(0)-n \mathbf{c}^{-1} \mathbf{I}_{\mathbf{k} k} \hat{\boldsymbol{\psi}}_{n-1}(s)\right], \\
& =\frac{\mathbf{B}_{0}^{*}(s)\left[{ }_{k} \boldsymbol{\psi}_{n}(0)-n \mathbf{c}^{-1} \mathbf{I}_{\mathbf{k} k} \hat{\boldsymbol{\psi}}_{n-1}(s)\right]}{\operatorname{Det}\left[\mathbf{B}_{0}(s)\right]}, \quad s>0,
\end{aligned}
$$


where $\mathbf{B}_{0}(s)=\left.\mathbf{B}(s)\right|_{\boldsymbol{\delta}=\mathbf{r}=\mathbf{0}, \mathbf{v}=\mathbf{I}}=s \mathbf{I}-\mathbf{c}^{-1}(\boldsymbol{\lambda}-\mathbf{A})+\mathbf{c}^{-1} \boldsymbol{\lambda} \hat{\mathbf{f}}(s)$ and $\mathbf{I}_{k}$ is an $m \times m$ diagonal matrix with the $k$-th element on the diagonal being 1 and all other elements on the diagonal being zero.

Using the same method as in deriving $\phi(0)$ in Section 3, we have two expressions for ${ }_{k} \boldsymbol{\psi}_{n}(0)$ :

$$
\begin{aligned}
{ }_{k} \boldsymbol{\psi}_{n}(0)= & n \int_{0}^{\infty} e^{-\boldsymbol{\Theta} x} \mathbf{c}^{-1} \mathbf{I}_{\mathbf{k} k} \boldsymbol{\psi}_{n-1}(x) d x, \quad n=1,2, \ldots, \\
{ }_{k} \boldsymbol{\psi}_{n}(0)= & n \mathbf{c}^{-1} \mathbf{I}_{\mathbf{k} k} \hat{\boldsymbol{\psi}}_{n-1}\left(\rho_{m}\right)+n\left(\mathbf{B}_{\mathbf{0}}^{*}\left[\rho_{1}, \rho_{2}, \ldots, \rho_{m}\right]\right)^{-1} \times \\
& \sum_{i=1}^{m-1} \mathbf{B}_{\mathbf{0}}^{*}\left[\rho_{1}, \ldots, \rho_{i}\right] \mathbf{c}^{-1} \mathbf{I}_{\mathbf{k} k} \hat{\boldsymbol{\psi}}_{n-1}\left[\rho_{i}, \ldots, \rho_{m}\right]
\end{aligned}
$$

where $\rho_{1}=0, \rho_{2}, \ldots, \rho_{m}$ are the roots of the generalized Lundberg equation and $\boldsymbol{\Theta}$ is the matrix defined in Section 4 with $\boldsymbol{\delta}=\mathbf{r}=\mathbf{0}$ and $\mathbf{v}=\mathbf{I}$.

It follows from Section 5 that $\left[\mathbf{B}_{0}(s)\right]^{-1}=\hat{\mathbf{W}}_{\mathbf{0 , 0}, \mathbf{I}}(s)$. Inverting Equation (6.1) yields for $n=1,2, \ldots$,

$$
{ }_{k} \boldsymbol{\psi}_{n}(u)=\mathbf{W}_{\mathbf{0 , 0}, \mathbf{I}}(u){ }_{k} \boldsymbol{\psi}_{n}(0)-n \int_{0}^{u} \mathbf{W}_{\mathbf{0 , 0}, \mathbf{I}}(u-x) \mathbf{c}^{-1} \mathbf{I}_{k}{ }_{k} \boldsymbol{\psi}_{n-1}(x) d x,
$$

where ${ }_{k} \boldsymbol{\psi}_{0}(u)=\boldsymbol{\psi}(u)$.

\section{Remarks}

1. Using similar arguments, we have

$$
\begin{aligned}
& \boldsymbol{\psi}_{n}(0)=n \int_{0}^{\infty} e^{-\boldsymbol{\Theta} x} \mathbf{c}^{-1} \boldsymbol{\psi}_{n-1}(x) d x \\
& \boldsymbol{\psi}_{n}(u)=\mathbf{W}_{\mathbf{0 , 0}, \mathbf{I}}(u) \boldsymbol{\psi}_{n}(0)-n \int_{0}^{u} \mathbf{W}_{\mathbf{0 , 0}, \mathbf{I}}(u-x) \mathbf{c}^{-1} \boldsymbol{\psi}_{n-1}(x) d x
\end{aligned}
$$

with $\boldsymbol{\psi}_{0}(u)=\boldsymbol{\psi}(u)$. See Li et al. (2015).

2. In particular, when $m=1$ the Markov-modulated risk model reduces to the classical risk model with $\mathbf{A}=\mathbf{\Theta}=0, W_{0,0,1}(u)=[1-\psi(u)] /[1-\psi(0)]$, and ${ }_{1} \boldsymbol{\psi}_{n}(u)=\psi_{n}(u)$. Then Equation (6.3) simplifies to

$$
\psi_{n}(0)=\frac{n}{c} \int_{0}^{\infty} \psi_{n-1}(u) d u
$$

and Equation (6.4) simplifies to the corresponding result in Lin and Willmot (2000). 


\section{$7 \quad$ Numerical illustrations}

Suppose again that there are two states $(m=2)$ with intensity matrix

$$
\mathbf{A}=\left(\begin{array}{cc}
-1 / 4 & 1 / 4 \\
3 / 4 & -3 / 4
\end{array}\right)
$$

with $\left(\pi_{1}, \pi_{2}\right)=(3 / 4,1 / 4)$. Further, as in Example 5.1, assume that $f_{1}(x)=$ $e^{-x}, f_{2}(x)=0.5 e^{-0.5 x}, x>0, \lambda_{1}=1, \lambda_{2}=2 / 3, c_{1}=4 / 3, c_{2}=5 / 3$ so that the positive loading condition holds true. Table 7.1 gives the expected ruin time, expected values and two types of covariance of durations of surplus in states 1 and 2 for varying $u$ and initial state $i=1$, where

$$
\operatorname{Cov}_{1}=\operatorname{Cov}\left(T_{u, 1} I\left(T_{u}<\infty\right), T_{u, 2} I\left(T_{u}<\infty\right)\right), \quad \operatorname{Cov}_{2}=\operatorname{Cov}\left(T_{u, 1}, T_{u, 2} \mid T_{u}<\infty\right) .
$$

\begin{tabular}{|c|c|r|r|r|r|}
\hline \multirow{2}{*}{$u$} & \multicolumn{5}{|c|}{$J(0)=1$} \\
\cline { 2 - 6 } & $\psi_{1}^{(1)}(u)$ & ${ }_{1} \psi_{1}^{(1)}(u)$ & ${ }_{2} \psi_{1}^{(1)}(u)$ & $\mathrm{Cov}_{1}$ & $\mathrm{Cov}_{2}$ \\
\hline 0 & 2.9078 & 2.2900 & 0.6178 & 27.31 & 34.65 \\
2 & 4.7390 & 3.6518 & 1.0872 & 52.94 & 94.37 \\
4 & 4.7820 & 3.8466 & 1.2104 & 64.76 & 156.87 \\
6 & 3.5313 & 3.5536 & 1.1628 & 68.34 & 222.70 \\
8 & 4.7820 & 3.0828 & 1.0384 & 66.71 & 292.41 \\
10 & 3.5313 & 2.5780 & 0.8876 & 61.94 & 366.40 \\
15 & 2.0407 & 1.5039 & 0.5368 & 44.83 & 571.61 \\
20 & 1.1096 & 0.8131 & 0.2965 & 28.72 & 806.92 \\
\hline
\end{tabular}

Table 7.1: Covariances and the expected values of durations in states 1 and 2

We can see in Table 7.1 that the expected times spent in states 1 and 2, given that $J(0)=1$, are increasing in $u$ at first, and are then decreasing in $u$ and converge to zero when $u$ goes to infinity. The unconditional covariance of the durations in states 1 and 2 is positive, increasing in $u$ at first and then decreasing in $u$, while the conditional covariance given ruin has occurred is an increasing function of $u$. Similar patterns hold for $J(0)=2$. Figure 7.1 shows the conditional expected durations in states 1 and 2 given that ruin has occurred for $J(0)=1$ (left) and $J(2)=2$ (right). The figure shows the conditional expected durations are increasing in $u$ for both $J(0)=1$ and $J(0)=2$, and so behave differently to the unconditional ones. Table 7.2 gives the expected values and two types of 

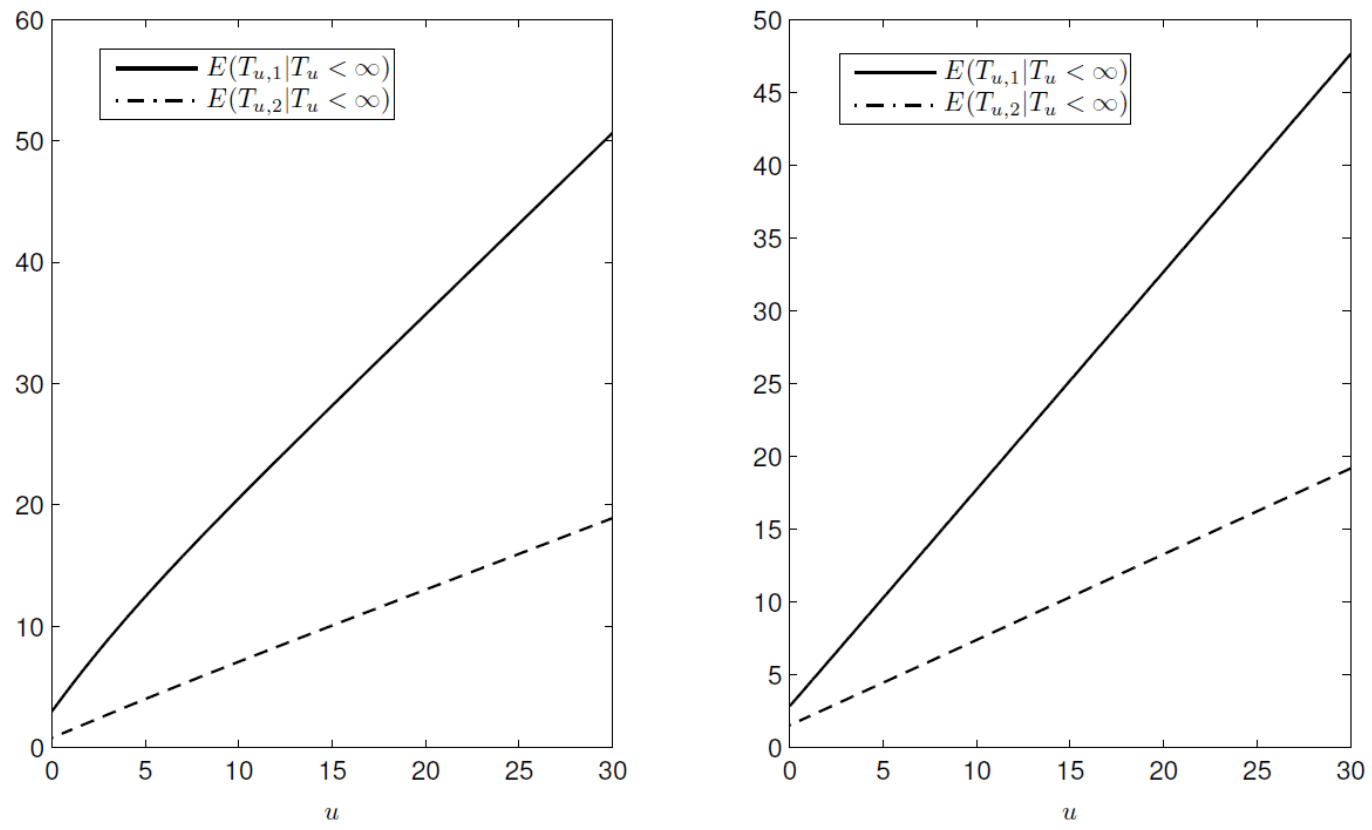

Figure 7.1: Expected times in states 1 and 2 given $T_{u}<\infty$ for $J(0)=1$ and $J(0)=2$

covariance of numbers of claims that occur in states 1 and 2 by the time of ruin for $J(0)=1$, where

$$
\begin{aligned}
\chi_{1}^{(1)}(u) & =\mathbb{E}\left[N_{u} I\left(T_{u}<\infty\right) \mid J(0)=1\right], \\
{ }_{k} \chi_{1}^{(1)}(u) & =\mathbb{E}\left[N_{u, k} I\left(T_{u}<\infty\right) \mid J(0)=1\right], \quad k=1,2, \\
\operatorname{Cov}_{3} & =\operatorname{Cov}\left(N_{u, 1} I\left(T_{u}<\infty\right), N_{u, 2} I\left(T_{u}<\infty\right)\right), \\
\operatorname{Cov}_{4} & =\operatorname{Cov}\left(N_{u, 1}, N_{u, 2} \mid T_{u}<\infty\right) .
\end{aligned}
$$

We can see that the expected numbers of claims in states 1 and 2 by the time of ruin, given that $J(0)=1$, are increasing in $u$ at first, then decreasing in $u$ and they converge to zero when $u$ goes to infinity. The unconditional covariance of the numbers of claims in states 1 and 2 is positive, increasing in $u$ at first and then decreasing in $u$, while the conditional covariance given ruin has occurred is strictly increasing in $u$. Similar patterns hold for $J(0)=2$. We can draw similar conclusions for aggregate amounts of claims in states 1 and 2 for both $J(0)=1$ and $J(0)=2$. 


\begin{tabular}{|c|c|r|r|r|r|}
\hline \multirow{2}{*}{$u$} & \multicolumn{5}{|c|}{$J(0)=1$} \\
\cline { 2 - 6 } & $\chi_{1}^{(1)}(u)$ & ${ }_{1} \chi_{1}^{(1)}(u)$ & ${ }_{2} \chi_{1}^{(1)}(u)$ & $\mathrm{Cov}_{3}$ & $\mathrm{Cov}_{4}$ \\
\hline 0 & 3.4450 & 2.8390 & 0.6060 & 23.77 & 29.98 \\
2 & 5.5376 & 4.4629 & 1.0747 & 47.77 & 83.00 \\
4 & 5.9003 & 4.6986 & 1.2017 & 60.27 & 139.50 \\
6 & 5.4849 & 4.3270 & 1.1579 & 65.34 & 200.39 \\
8 & 4.7823 & 3.7459 & 1.0364 & 65.25 & 266.32 \\
10 & 4.0155 & 3.1279 & 0.8875 & 61.74 & 337.72 \\
20 & 1.2812 & 0.9834 & 0.2978 & 30.37 & 781.77 \\
\hline
\end{tabular}

Table 7.2: Covariances and the expected numbers of claims in states 1 and 2 for $i=1$

\section{Quantities related to the hitting time of an upper barrier}

For $u \leq b$, define $T_{u}^{b}=\min \{t \geq 0: U(t)=b\}$ to be the first time when the surplus reaches level $b$. Let $N_{u}^{b}:=N\left(T_{u}^{b}\right)$ be the number of claims and $S_{u}^{b}:=S\left(T_{u}^{b}\right)=$ $\sum_{n=1}^{N_{u}^{b}} X_{n}$ be the aggregate claims by time $T_{u}^{b}$, respectively. Further, $T_{u}^{b}, N_{u}^{b}$ and $S_{u}^{b}$ can be decomposed as

$$
T_{u}^{b}=\sum_{k=1}^{m} T_{u, k}^{b}, \quad N_{u}^{b}=\sum_{k=1}^{m} N_{u, k}^{b}, \quad S_{u}^{b}=\sum_{k=1}^{m} S_{u, k}^{b},
$$

where $T_{u, k}^{b}$ is the duration in state $k \in E, N_{u, k}^{b}$ is the number of claims that occur in state $k$, and $S_{u, k}^{b}$ is the aggregate claims that occur in state $k$, until the time $T_{u}^{b}$. For $\delta_{k}, r_{k} \geq 0,0<v_{k} \leq 1, k \in E$, define

$$
\tau_{i, j}(u ; b)=\mathbb{E}\left[e^{-\sum_{k=1}^{m}\left(\delta_{k} T_{u, k}^{b}+r_{k} S_{u, k}^{b}\right)} \prod_{k=1}^{m} v_{k}^{N_{u, k}^{b}} I\left(J\left(T_{u}^{b}\right)=j\right) \mid J(0)=i\right],
$$

for $u \leq b$, with $\tau_{i, j}(b ; b)=I(i=j)$.

Let $\boldsymbol{\tau}(u ; b)=\left(\tau_{i, j}(u ; b)\right)_{i, j=1}^{m}$. Using the same arguments as in Section 3, we have for $u \leq b$ :

$$
\boldsymbol{\tau}^{\prime}(u ; b)=\mathbf{c}^{-1}(\boldsymbol{\lambda}+\boldsymbol{\delta}-\mathbf{A}) \boldsymbol{\tau}(u ; b)-\mathbf{c}^{-1} \boldsymbol{\lambda} \mathbf{v} \int_{0}^{\infty} e^{-\mathbf{r} x} \mathbf{f}(x) \boldsymbol{\tau}(u-x ; b) d x,
$$

with $\boldsymbol{\tau}(b ; b)=\mathbf{I}$. 
From the facts that (i) $\boldsymbol{\tau}(b ; b)=\mathbf{I}$; (ii) $\boldsymbol{\tau}(u ; b)$ is a function of $(b-u)$; (iii) $\boldsymbol{\tau}\left(u ; b_{1}\right) \boldsymbol{\tau}\left(b_{1} ; b\right)=\boldsymbol{\tau}(u ; b)$ for $u \leq b_{1} \leq b$, we conclude that $\boldsymbol{\tau}(u ; b)$ must have the following form:

$$
\boldsymbol{\tau}(u ; b)=e^{-\boldsymbol{\Gamma}(b-u)}, \quad u \leq b,
$$

where $\Gamma$ is an $m \times m$ matrix and all of its eigenvalues must have positive real parts. Substituting Equation (8.3) into Equation (8.2) shows that $\boldsymbol{\Gamma}$ satisfies

$$
\boldsymbol{\Gamma}+\mathbf{c}^{-1} \boldsymbol{\lambda} \mathbf{v} \int_{0}^{\infty} \mathbf{f}(x) e^{-\mathbf{r} x} e^{-\boldsymbol{\Gamma} x} d x-\mathbf{c}^{-1}(\boldsymbol{\lambda}+\boldsymbol{\delta}-\mathbf{A})=\mathbf{0}
$$

In Section 3, we have shown that $\operatorname{Det}[\mathbf{B}(s)]=0$ has $m$ roots $\rho_{1}, \rho_{2}, \ldots, \rho_{m}$, where

$$
\mathbf{B}(s)=s \mathbf{I}-\mathbf{c}^{-1}(\boldsymbol{\lambda}+\boldsymbol{\delta}-\mathbf{A})+\mathbf{c}^{-1} \boldsymbol{\lambda} \mathbf{v} \int_{0}^{\infty} \mathbf{f}(x) e^{-(\mathbf{r}+s \mathbf{I}) x} d x .
$$

For $i=1,2, \ldots, m$, let column vector $\overrightarrow{\mathbf{h}}_{i}^{\top}$ be an eigenvector of $\mathbf{B}\left(\rho_{i}\right)$ corresponding to the eigenvalue 0 . Then

$$
\overrightarrow{\mathbf{0}}^{\top}=\mathbf{B}\left(\rho_{i}\right) \overrightarrow{\mathbf{h}}_{i}^{\top}=\rho_{i} \overrightarrow{\mathbf{h}}_{i}^{\top}+\mathbf{c}^{-1} \boldsymbol{\lambda} \mathbf{v} \int_{0}^{\infty} \mathbf{f}(x) e^{-\mathbf{r} x} e^{-\rho_{i} x} \overrightarrow{\mathbf{h}}_{i}^{\top} d x-\mathbf{c}^{-1}(\boldsymbol{\lambda}+\boldsymbol{\delta}-\mathbf{A}) \overrightarrow{\mathbf{h}}_{i}^{\top} .
$$

In matrix notation, we have

$$
\mathbf{H} \boldsymbol{\rho}+\mathbf{c}^{-1} \boldsymbol{\lambda} \mathbf{v} \int_{0}^{\infty} \mathbf{f}(x) e^{-\mathbf{r} x} \mathbf{H} e^{-\boldsymbol{\rho} x} d x-\mathbf{c}^{-1}(\boldsymbol{\lambda}+\boldsymbol{\delta}-\mathbf{A}) \mathbf{H}=\mathbf{0},
$$

where $\mathbf{H}=\left(\overrightarrow{\mathbf{h}}_{1}^{\top}, \overrightarrow{\mathbf{h}}_{2}^{\top}, \ldots, \overrightarrow{\mathbf{h}}_{m}^{\top}\right)$ and $\boldsymbol{\rho}=\operatorname{diag}\left(\rho_{1}, \rho_{2}, \ldots, \rho_{m}\right)$. As we assume that $\rho_{1}, \rho_{2}, \ldots, \rho_{m}$ are distinct, $\overrightarrow{\mathbf{h}}_{1}^{\top}, \overrightarrow{\mathbf{h}}_{2}^{\top}, \ldots, \overrightarrow{\mathbf{h}}_{m}^{\top}$ are linearly independent and so $\mathbf{H}$ is invertible. Multiplying both sides of Equation (8.5) by $\mathbf{H}^{-1}$, we obtain

$$
\mathbf{H} \boldsymbol{\rho} \mathbf{H}^{-1}+\mathbf{c}^{-1} \boldsymbol{\lambda} \mathbf{v} \int_{0}^{\infty} \mathbf{f}(x) e^{-\mathbf{r} x} \mathbf{H} e^{-\boldsymbol{\rho} x} \mathbf{H}^{-1} d x-\mathbf{c}^{-1}(\boldsymbol{\lambda}+\boldsymbol{\delta}-\mathbf{A})=\mathbf{0} .
$$

Comparing Equation (8.4) with Equation (8.6), we obtain

$$
\Gamma=\mathbf{H} \rho \mathbf{H}^{-1} \text {. }
$$

\section{Remarks}

1. When $\delta_{i}=\delta, r_{i}=r$ and $v_{i}=v$ for $i=1,2, \ldots, m$, we have $\boldsymbol{\tau}(u ; b)=$ $\left(\tau_{i, j}(u ; b)\right)_{i, j=1}^{m}=e^{-\boldsymbol{\Gamma}(b-u)}$, where

$$
\tau_{i, j}(u ; b)=\mathbb{E}\left[e^{-\delta T_{u}^{b}-r S_{u}^{b}} v^{N_{u}^{b}} I\left(J\left(T_{u}^{b}\right)=j \mid J(0)=i\right], \quad u \leq b .\right.
$$


2. When $m=1$, the Markov-modulated risk model simplifies to the classical risk model, $\boldsymbol{\Gamma}$ simplifies to $\rho$ and the matrix $\boldsymbol{\tau}(u ; b)$ simplifies to a scalar $\tau(u ; b)$ with

$$
\tau(u ; b)=\mathbb{E}\left[e^{-\delta T_{u}^{b}-r S_{u}^{b}} v^{N_{u}^{b}}\right]=e^{-\rho(b-u)}, \quad u \leq b,
$$

where $\rho:=\rho(\delta, r, v)>0$ satisfies the following equation:

$$
c s-(\lambda+\delta)+\lambda v \hat{f}(r+s)=0 .
$$

3. The matrix $\Theta$ in Section 4 is different from the matrix $\boldsymbol{\Gamma}$, as it can be shown that $\Theta$ satisfies the following matrix equation:

$$
\boldsymbol{\Theta}+\int_{0}^{\infty} e^{-\boldsymbol{\Theta} x} e^{-\mathbf{r} x} \mathbf{f}(x) d x \mathbf{c}^{-1} \boldsymbol{\lambda} \mathbf{v}-\mathbf{c}^{-1}(\boldsymbol{\lambda}+\boldsymbol{\delta}-\mathbf{A})=\mathbf{0},
$$

while matrix $\boldsymbol{\Gamma}$ satisfies a different equation, as in (8.4).

\section{Concluding remarks}

This paper shows how quantities such as duration, number of claims, and aggregate claims in a certain state by the time of ruin or by the first passage time of a certain level, can be analyzed through a generalized Gerber-Shiu function. The joint transform of the duration of surplus, number of claims, and the aggregate claims in a certain state by the two-sided first exit time can be analyzed accordingly. Some results and methods can be extended to the MAP (Markovian Arrival Process) risk model.

\section{References}

[1] Asmussen, S. (1989) Risk theory in a Markovian environment. Scandinavian Actuarial Journal, 69-100.

[2] Asmussen, S., Frey, A., Rolski, T. and Schmidt V. (1995) Does Markovmodulation increase the risk? ASTIN Bulletin 25, 49-66.

[3] Bäuerle, N. (1996) Some results about the expected ruin time in Markovmodulated risk models. Insurance: Mathematics $\&$ Economics 18, 119-127.

[4] De Smit, J. (1983) The queue GI/M/s with customers of different types or the queue GI/Hm/s. Advances in Applied Probability 15, 392-419. 
[5] Dickson, D.C.M. and Hipp, C. (2001) On the time to ruin for Erlang(2) risk process. Insurance: Mathematics 83 Economics 29, 333-344.

[6] Feng, R. (2009) A matrix operator approach to the analysis of ruin-related quantities in the phase-type renewal risk model. Schweizerische Aktuarvereinigung Mitteilungen 1, 71-87.

[7] Fischer, W. and Meier-Hellstern, K. (1993) The Markov-modulated Poisson process (MMPP) cookbook. Performance Evaluation 18, 149-171.

[8] Gerber, H.U. and Shiu, E.S.W. (2005) The time value of ruin in a Sparre Andersen model. North American Actuarial Journal 9(2), 49-69.

[9] Higham, N.J. and Kim, H.-M. (2000) Numerical analysis of a quadratic matrix equation. IMA Journal of Numerical Analysis 20, 499-519.

[10] Kyprianou, A.E. (2013) Gerber-Shiu Risk Theory. Springer.

[11] Li, J., Dickson, D.C.M. and Li, S. (2015) Some ruin problems for the MAP risk model. Insurance: Mathematics $\&$ Economics 65, 1-8

[12] Li, S. and Garrido, J. (2004) On ruin for the $\operatorname{Erlang}(n)$ risk process. Insurance: Mathematics \&6 Economics 34, 391-408.

[13] Li, S. and Lu, Y. (2007) Moments of the dividend payments and related problems in a Markov-modulated risk model. North American Actuarial Journal 11(2), 65-76.

[14] Li, S. and Lu, Y. (2008) The decompositions of the discounted penalty functions and dividends-penalty identity in a Markov-modulated risk model. ASTIN Bulletin 38, 53-71.

[15] Li, S. and Ren, J. (2013) The time of recovery and the maximum severity of ruin in a perturbed MAP risk process. Statistics and Probability Letters 83(4), 993-998.

[16] Lin, X.S. and Willmot, G.E. (2000) The moments of the time of ruin, the surplus before ruin, and the deficit at ruin. Insurance: Mathematics \& Economics 27, 19-44.

[17] Lu, Y. (2006) On the severity of ruin in a Markov-modulated risk model. Scandinavian Actuarial Journal, 183-202. 
[18] Lu, Y. and Li, S. (2005) On the probability of ruin in a Markov-modulated risk model. Insurance: Mathematics 85 Economics 37, 522-532.

[19] Ng, A.C.Y. and Yang, H. (2006) On the joint distribution of surplus before and after ruin under a Markovian regime switching model. Stochastic Processes and their Applications 116, 244-266.

[20] Ren, J. (2007) The discounted joint distribution of the surplus prior to ruin and the deficit at ruin in a Sparre Andersen model. North American Actuarial Journal 11(3), 128-136.

[21] Schmidli, H. (1997) Estimation of the Lundberg coefficient for a Markov modulated risk model. Scandinavian Actuarial Journal, 48-57.

[22] Snoussi, M. (2002) The severity of ruin in Markov-modulated risk models. Bulletin of the Swiss Association of Actuaries, 31-43. 


\section{University Library}

\section{- M M N E R VA A gateway to Melbourne's research publications}

Minerva Access is the Institutional Repository of The University of Melbourne

Author/s:

Li, J;Dickson, DCM;Li, S

Title:

Analysis of some ruin-related quantities in a Markov-modulated risk model

Date:

2016

Citation:

Li, J., Dickson, D. C. M. \& Li, S. (2016). Analysis of some ruin-related quantities in a Markov-modulated risk model. Stochastic Models, 32 (3), pp.351-365. https:// doi.org/10.1080/15326349.2015.1121399.

Persistent Link:

http://hdl.handle.net/11343/120635 